\title{
Quality Prediction of DWT-Based Compression for Remote Sensing Image Using Multiscale and Multilevel Differences Assessment Metric
}

\author{
Hongxu Jiang, ${ }^{1,2}$ Kai Yang, ${ }^{3}$ Tingshan Liu, ${ }^{1,2}$ and Yongfei Zhang ${ }^{1,2}$ \\ ${ }^{1}$ Beijing Key Laboratory of Digital Media, School of Computer Science and Engineering, Beihang University, Beijing 100191, China \\ ${ }^{2}$ State Key Laboratory of Virtual Reality Technology and Systems, Beihang University, Beijing 100191, China \\ ${ }^{3}$ Institute of Computing Technologies, China Academy of Railway Sciences, Beijing 100081, China
}

Correspondence should be addressed to Hongxu Jiang; jianghx@buaa.edu.cn

Received 27 December 2013; Revised 30 April 2014; Accepted 3 June 2014; Published 29 June 2014

Academic Editor: Claudio R. Fuerte-Esquivel

Copyright (C) 2014 Hongxu Jiang et al. This is an open access article distributed under the Creative Commons Attribution License, which permits unrestricted use, distribution, and reproduction in any medium, provided the original work is properly cited.

\begin{abstract}
Accurate assessment and prediction of visual quality are of fundamental importance to lossy compression of remote sensing image, since it is not only a basic indicator of coding performance, but also an important guide to optimize the coding procedure. In the paper, a novel quality prediction model based on multiscale and multilevel distortion (MSMLD) assessment metric is preferred for DWT-based coding of remote sensing image. Firstly, we propose an image quality assessment metric named MSMLD, which assesses quality by calculating distortions in three levels and multiscale sampling between original images and compressed images. The MSMLD method not only has a better consistency with subjective perception values, but also shows the distortion features and visual quality of compressed image well. Secondly, some significant characteristics in spatial and wavelet domain that link well with quality criteria of MSMLD are chosen with multiple linear regression and used to establish a compression quality prediction model of MSMLD. Finally, the quality prediction model is extended to a wider range of compression ratios from $4: 1$ to $20: 1$ and tested with experiment. The experimental results show that the prediction accuracy of the proposed model is up to $98.33 \%$, and its mean prediction error is less than state-of-the-art methods.
\end{abstract}

\section{Introduction}

During the past few decades, several kinds of transforms have been developed for image compression [1], such as Karhunen-Loève transform (KLT), discrete cosine transform (DCT), and discrete wavelet transform (DWT). JPEG [2] and other DCT-based coding techniques [3] have been put to use in many space missions, such as Clementine, TRACE, and SPOT-5 [4]. DWT offers a highly efficient and flexible way for subband decomposition of image, and it is a very powerful tool for image coding. DWT is suggested in JPEG2000 [57], which provides numerous advantages over the JPEG. As one of DWT-based coding methods, JPEG2000 has been applied to remote sensing image compression widely. For example, in these satellites such as BilSAT-1, RASAT, and $\mathrm{X}$-SAT, all image compression payloads are designed with JPEG2000 [4]. JPEG2000 is also used for the other remote sensing image compression referred to in $[8,9]$. In addition, The Consultative Committee for Space Data Systems (CCDS) published a recommended standard, CCSDS-IDC Blue Book [10], which establishes a data compression algorithm consisting of DWT and bit-plane encoder. Although there are so many methods for remote sensing image coding, obviously, DWT-based methods are dominant in practical applications in recent years.

Predicting image quality at a preencoding stage or without coding is very useful for image compression, especially for DWT-based coding algorithms [11], for it can be applied to bit allocation, filter bank selection, coding scheme selection, encoding parameter decision, and so forth. PSNR-based quality criteria are most commonly used in quality prediction for wavelet coding $[12,13]$. They can predict the compression quality without coding, only by constructing a function between image characteristics and 
the quality values. Although existing PSNR-based quality prediction methods allow us to evaluate the numerical difference between images for DWT coding, they have two defects when being applied to remote sensing image as follows. Firstly, images from different categories (natural, remote sensing, synthetic, compound, medical, etc.) tend to show different spatial domain characteristics, but the existing models are mainly designed and tested with nature scene images, instead of remote sensing images. Secondly, the PSNR-based compression quality prediction methods are not consistent with the human vision system (HVS) and do not reflect the appreciation of the humane eye accurately. Thus it is interesting to integrate other quality criteria which have a good consistency with subjective perception values.

In the paper, a quality assessment method is preferred primarily for remote sensing image compression, basing on multiscale and multilevel difference (MSMLD), which has a good consistency with subjective perception values, and the objective assessment results can well reflect the visual quality of remote sensing compressed images. After that, by analyzing the correlation between MSMLD quality criteria and image characteristics in spatial domain and wavelet domain, a quality prediction model based on MSMLD is designed for DWT coding algorithms. The rest of this paper is organized as follows. In the next section, we will review previous works on image compression quality prediction. Section 3 presents our objective image quality assessment metrics called MSMLD for remote sensing image. In Section 4 , a quality prediction model based on MSMLD is preferred and implemented for DWT-based compression. In Section 5, our proposed method is compared with notable existing works. Finally, the conclusions and future work are drawn in Section 6.

\section{Related Works}

An essential aspect of quality prediction is to establish a function between image inherent characteristics and quality assessment criteria; thus only by calculating some values associated with image characteristics can the final image quality be predicted accurately at a preencoding stage or without coding, instead of after decoding and assessment. It is very important and advantageous for image compression to foresee the image quality accurately during coding because that makes it impossible to adjust algorithm promptly to get better coding performance with less computational cost. Currently for image compression, PSNR-based methods are most widely used to measure and predict image compression quality at a preencoding stage or without coding and also be a focus of research. For example, Koumaras et al. [14] predicted the compression distortion of DCT using the variances of image. Kourzi et al. [12] achieved the prediction by constructing wavelet filter space and frequency index. Saha and Vemuri [13] chose image activity measure (IAM) as prediction factor and established the IAM-PSNR equation, basing on analyzing the relevance between the inherent characteristics of the images and coding performance; it was special for the compression algorithm based on wavelet transform. On the basis of this, Li and Wang [11] analyzed the dependency between IAM and PSNR in different functional forms and constructed a prediction model of PSNR based on IAM, so that the prediction accuracy could be improved. In all of these methods, the correlations between image inherent characteristics and image quality assessment are thoughtfully analyzed and widely applied, and most of the research and applications are centered on ordinary scene images instead of remote sensing images. However, images from different categories tend to show different spatial domain characteristics, and there are many differences in correlations between image inherent characteristics and image distortion assessment for ordinary scene images and remote sensing images. Wei et al. [15] studied the problem of distortion prediction for remote sensing image compression by analyzing image characteristics in spatial domain and wavelet domain, and some image characteristics significantly related to PSNR were selected and used to establish a distortion prediction model with multiple linear regression to achieve the prediction of image distortion during coding. However, in [15] the selection of image characteristics is too dependent on the empirical values, and furthermore PSNR is used as the indicator of distortion prediction, whose ability to evaluate the image quality is relatively limited. So the method in [15] has inherent defects in distortion assessment and prediction for remote sensing image compression.

Image quality evaluation methods can be divided into two classes: subjective assessments and objective assessments. Due to the observing abilities, display conditions, and scoring standards, the subjective assessments by human are prone to be inconvenient, time-consuming, and unstable. The objective assessments propose a judgment on image quality by simulating the human vision system, by algorithms designed to imitate human subjectivity with a formulation. The objective methods include three kinds, such as full-reference methods, part-reference methods, and no-reference methods. Fullreference methods are the foundation of other types and outperform the other two types. The notable full-reference quality metrics are usually classified into three categories as follows [16]. (1) The first category is mathematically convenient metrics; PSNR and MSE are simply formulated and hence are computationally efficient; they are widely used in remote sensing image compression for a long time [17-19]. However, this kind of metric has a congenital defect where it provides an inaccurate representation of the image quality, and its evaluation result usually has deviation from subjective perception values in a way. (2) The second category is near-threshold psychophysics based metrics. Taking WSNR [20] and NQM [21] for example, these metrics attempt to simulate the perception of image distortion by analysis of human vision sensitivity. However, these metrics confine to visual research limitations. What is more important is that the existing research works mostly focus on the feature analysis and evaluation of natural scenery images, which are quite different from remote sensing images for their unique characteristics. (3) The third category is metrics based on overall perception. These metrics avoid simulating the complex human vision but measuring the structural distortions of images. Under the assumption that human visual perception is highly adapted for extracting structural information from a 
scene, structural similarity (SSIM) [22] between the distorted and original images was proposed to assess image quality, and it was further extended to the multiscale SSIM (MSSSIM) [23] by sliding windows. Recently, VIF was proposed [24] to adopt a novel visual information fidelity criterion that quantified the Shannon information presented in the distorted image relative to the information presented in the original image. According to [24], VIF outperforms other quality metrics. As those structure based metrics formulate the assessment functions on the higher regional information rather than pixels, they are more consistent with the HVS but insufficient in evaluating small target distortions.

Although there are plenty of image quality evaluation methods, they are mainly common-oriented for ordinary image test and application. For example, the recently proposed SSIM and VIF are typically common-oriented and state-of-the-art methods and are adopted and used in many applications. Because the remote sensing images are quite different from ordinary images in acquisition mode and image content, the evaluation results often deviate from the subjective interpretation in a way when these common assessment methods are used for remote sensing image compression quality evaluation. Relatively, there are a small number of algorithms devoted to the evaluation of remote sensing image compression and in which distortion characteristics of remote sensing image compression are taken into account partly. Moreover, these methods are mainly focused on low-level image features, such as pixel differences, image variance, and image histogram, using less high-level features, such as image structure and image content [25]. In overall performance term, these specific methods are still behind the SSIM, VIF, and so forth. Therefore, in this paper we propose an image quality metric to reveal image compression distortions and perform more comprehensive evaluation ability for different size distortions of remote sensing image compression.

Above all, in order to get a novel compression quality prediction model that has a good consistency with subjective perception values and for the prediction results to accurately reflect the visual quality of remote sensing compressed images, it is primarily necessary to study a novel image quality assessment metric according to the inherent characteristics of the remote sensing image; then, basing on the novel metric, a prediction model should be established for DWTbased compression to predict image compression quality at a preencoding stage or without coding.

\section{Image Quality Assessment Metric Based on Multilevel Difference}

3.1. Image Distortion Analysis Based on Multilevel Difference. Images from different types (natural, remote sensing, synthetic, compound, medical, etc.) tend to show different spatial domain characteristics. For example, when a variety of images of different categories are compressed using a fixed wavelet filter and a given compression ratio, it is observed that the PSNR values vary widely as much as dozens of $\mathrm{dB}$ from image to image [13]. The HVS research shows that when a remote sensing image is being observed, the perceptual information is not only equal to the information entropy of the pixel, but also affected by its position and surrounding pixels. The information of a pixel not only is correlated with its self-luminance, but also can be significantly affected by its neighbor pixels as shown in Figure 1. Due to the different neighbor pixels, the identical pixel $i$ is perceived as with different luminance values.

Optical remote sensing images, especially high resolution ones, are often characterized with typical features, such as numerous small targets, complex edge texture, and uneven distribution of image content. After lossy compression, some image distortions like loss of target, texture blurring, edge jitter, and block effect may occur. As shown in Figure 2, when analyzing and assessing remote sensing images, a hierarchical difference can be used to reflect the different aspects of image distortions. For example, the pixel changes can reflect the loss on small targets in images; the regional difference can reflect the distorted edge and fuzzy texture in images; the difference of image content can reflect the classification of image characteristics. Finally, the image quality can be comprehensively evaluated by all these differences. Because the current image quality evaluation methods cannot effectively take into account the distortion of different levels and scales, there are some limitations in evaluation results, and it is hard to get a good consistency with subjective perception. Therefore, it is the key of research for remote sensing image compression assessment that how to comprehensively descript the image distortion at different levels and scales will make objective evaluation results be closer to the subjective interpretation.

Based on the above, image quality assessment should be comprehensive descriptions of self-luminance, contexture impact, and pixel feature. In this paper, an image quality assessment method based on multilevel distortions (MLD) is designed to get better consistency with subjective perception values; in the proposed MLD method, differences in pixels, neighborhood, and structure between the original image and the decompressed image are calculated to reveal their inherent relationship with subjective interpretation. The overall flow of the proposed MLD includes three steps. First, all pixels of the original image and the distorted image are classified into three types according to image features. Second, the difference between original image and distorted image is calculated in point level and neighborhood level for each pixel. Finally, evaluation result of MLD is obtained by calculating the weighted distortion for each image pixel.

In the next section, the three levels of distortions will be formulated firstly, and the assessment metrics promoted to multiscale will be presented subsequently.

\subsection{Calculation of Multilevel Differences}

3.2.1. Calculating the Differences of Images. Let $X=\left\{x_{i} \mid\right.$ $i=1,2, \ldots, M\}$ and $Y=\left\{y_{i} \mid i=1,2, \ldots, M\right\}$ represent 


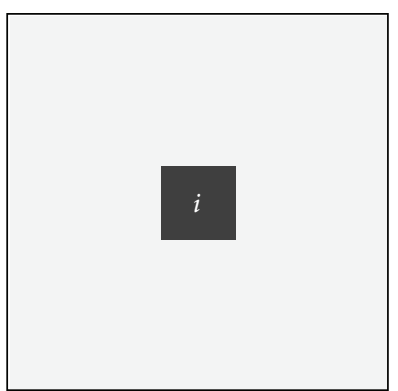

(a)

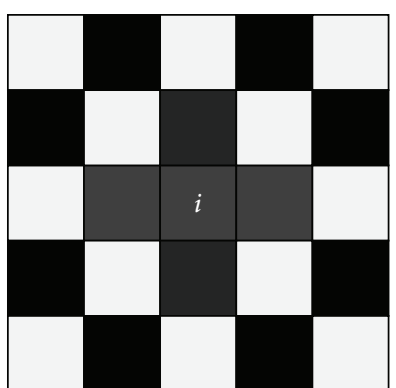

(b)

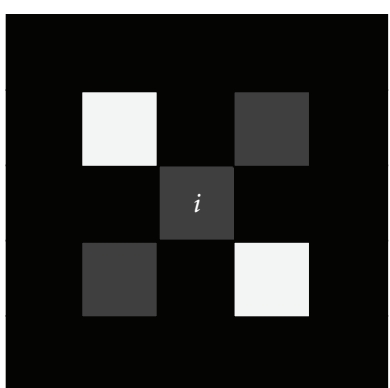

(c)

FIGURE 1: Visual difference of subjective interpretation with different contextures.

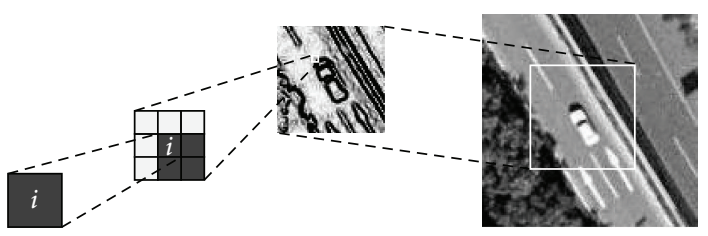

FIgURE 2: Appearance of image in different level and contextures.

the original image and its distorted image, respectively; $x_{i}$ and $y_{i}$ represent the luminance value of pixel $i$ in the original and distorted image. The pixel-level distortion $p\left(x_{i}, y_{i}\right)$ and the contexture-level distortion $n\left(x_{i}, y_{i}\right)$ are defined, respectively, as

$$
\begin{gathered}
p\left(x_{i}, y_{i}\right)=\frac{\left(x_{i}-y_{i}\right)^{2}}{x_{i}^{2}+y_{i}^{2}} \approx 1-\frac{2 x_{i} y_{i}}{x_{i}^{2}+y_{i}^{2}+\varepsilon}, \\
n\left(x_{i}, y_{i}\right)=\frac{\left(D_{x_{i}}-D_{y_{i}}\right)^{2}}{D_{x_{i}}^{2}+D_{y_{i}}^{2}+\varepsilon} \approx 1-\frac{2 D_{x_{i}} D_{y_{i}}}{D_{x_{i}}^{2}+D_{y_{i}}^{2}+\varepsilon},
\end{gathered}
$$

where $\varepsilon(\varepsilon \rightarrow 0)$ is a nonzero constant to keep the divisor unequal to zero, and in the paper $\varepsilon$ is set as 0.01 according to other parameters. $D_{x_{i}}$ is the contexture impact of pixel $i$ in the original image $X ; D_{y_{i}}$ is the contexture impact of pixel $i$ in the distorted image $Y$. They are defined as

$$
\begin{aligned}
& D_{x_{i}}=\sum_{j=1}^{N} \omega_{j}\left|x_{j}-x_{i}\right|, \\
& D_{y_{i}}=\sum_{j=1}^{N} \omega_{j}\left|y_{j}-y_{i}\right|,
\end{aligned}
$$

where $x_{j}(j \in[1, N])$ are the contexture pixels of $x_{i}$. $y_{j}(j \in[1, N])$ are the contexture pixels of $y_{i} . \omega=$ $\left\{\omega_{j} \mid j=1,2, \ldots, N\right\}$ is the weight set generated by the $7 \times 7$ circular-symmetric Gaussian weighting function with standard deviation of 1.5 samples.

3.2.2. Classifying the Pixels of Images. For observing remote sensing image, human pays more attention to two factors; one is the mutation position in image and the other is the distortion in mutation position. Therefore, the different weight should be set to the different distortion position as assessing image quality. Therefore, in order to assess image distortion accurately, all pixels should be divided into variation pixels and smooth pixels. Variation pixels are pixels with large gradient magnitude, such as the target edge pixels, textures pixels, and boundary pixels. Smooth pixels are pixels with small gradient magnitude. In the paper all pixels are classified into smooth-maintain pixels and variation-maintain pixels, according to Sobel gradient magnitudes with the threshold of $\beta$. The value of $\beta$ is optimized by objective experiments, as shown in Figure 3. In the figure, the number of variationmaintain pixels is gradually reduced with the increase of $\beta$, and in order to make the variation pixels close to subject variation interpretation, the value of $\beta$ is set to 0.02 as a tradeoff.

The procedure of classifying pixels of images can be summarized as follows.

(1) Calculate the gradient magnitudes of the original image $X$ and its distorted image $Y$ with Sobel operator, respectively.

(2) Set the classifying threshold value $\beta$ (here $\beta$ is 0.02 ) and classify the pixels into the smooth pixel sets $X_{s}$ and $Y_{s}$ and the variation pixel sets $X_{E}$ and $Y_{E}$ according to $\beta$. Pixels in $X_{s}$ and $Y_{s}$ are pixels with small gradient magnitude that is less than $\beta$. Pixels in $X_{E}$ and $Y_{E}$ are pixels with large gradient magnitude that is not less than $\beta$.

(3) Smooth pixels and variation pixels are refined and divided further, based on the following definitions. 


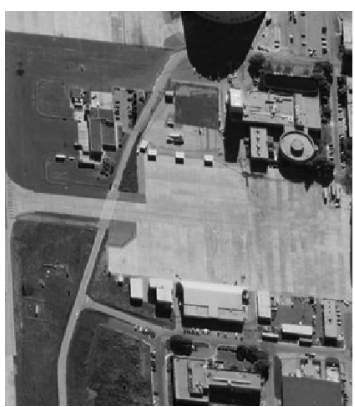

(a)

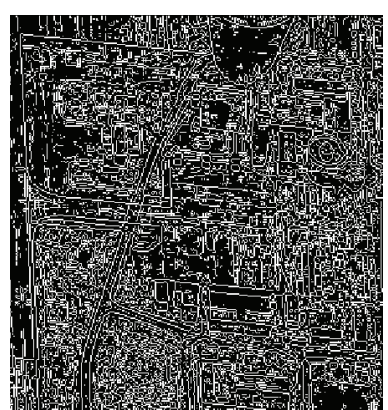

(b)

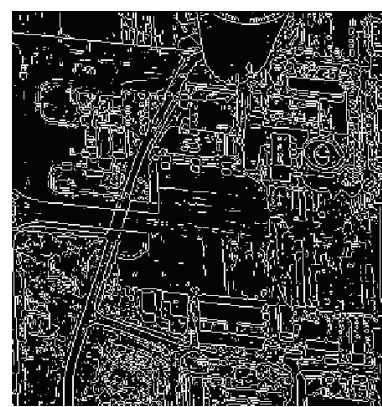

(c)

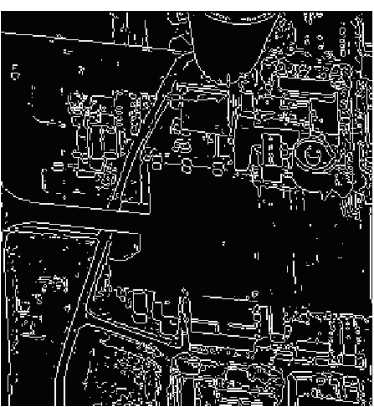

(d)

Figure 3: Classifying pixels according to gradient magnitudes with different threshold. (a) The original image; (b) $\beta=0.01$; (c) $\beta=0.02$; (d) $\beta=0.04$

If $x_{i} \in X_{S} \& y_{i} \in Y_{S}$, pixel $i$ is classified as a smooth-maintain pixel.

Else if $x_{i} \in X_{V} \& y_{i} \in Y_{V}$, pixel $i$ is classified as a variation-maintain pixel.

Else pixel $i$ is classified as a feature-changed pixel.

3.2.3. Calculating the Comprehensive Evaluation. In order to get a comprehensive evaluation of remote sensing image compression, an integral calculation of multilevel distortions is necessary after pixel classifying. The procedure of calculating the comprehensive evaluation can be summarized as follows.

(1) Classify each pixel $i(i=1,2, \ldots, M)$ in the original image $X$ and distorted image $Y$ into smooth-maintain pixel, variation-maintain pixel, or feature-changed pixel.

(2) Calculate the evaluation weight $w_{i}$ of content-level distortion, and it is set as the ratio of smooth pixel number to variation pixel number.

(3) Calculate $p\left(x_{i}, y_{i}\right)$ and $n\left(x_{i}, y_{i}\right)$ for each pixel $i$, respectively.

(4) Calculate the final image quality evaluation according to formula (3). Consider

$$
\operatorname{MLD}(X, Y)=\sum_{i=1}^{M} w_{i} p\left(x_{i}, y_{i}\right) n\left(x_{i}, y_{i}\right) .
$$

3.3. Multiscale Promotion of MLD. The perception of image details depends on the sampling density of image signals, the observation distance, and the observer's visual perceptual capability. According to Wang et al.s method [23], the proposed MLD is promoted to multiscale metrics named MSMLD for rough granulation applications. We extend our MLD to the multiscale metrics MMLD for rough granulation applications, as shown in Figure 4. In MSMLD the impacts of varying image resolutions and view conditions are well considered. The main idea is to apply the single-scale MLD on the sampled images of the original images and distorted images.
The procedure of MSMLD can be summarized as follows.

(1) In light of image size, the number of image downsampling $K$ is calculated by setting $K=\min ([W / 256],[H / 256])$, where $W$ and $H$ are the width and height of the image, respectively.

(2) $L$ is a set whose members are the mean filter factors $l$, and it is defined as $L=\{0,1, \ldots, K\}$. Its members are determined by $K$; for example, $L=\{0\}$ when $K$ is 0 , $L=\{0,1\}$ when $K$ is $1, L=\{0,1,2,3\}$ when $K$ is 3 , and so forth.

(3) For every element $l$ of set $L$, the original image $X$ and distorted image $Y$ are filtered and downsampled, using mean filter with window size of $2^{l} \times 2^{l}$, and $X^{l}$ and $Y^{l}$ are got separately; the width and height of the images $X^{l}$ and $Y^{l}$ are downsampled into $W / 2^{l}$ and $H / 2^{l}$.

(4) Based on the steps in Section 3.2.3, the final image quality evaluation $\operatorname{MLD}\left(X^{l}, Y^{l}\right)$ is calculated for $X^{l}$ and $Y^{l}$ according to (3).

(5) Iteratively compute steps (3) and (4) until there is an $\operatorname{MLD}\left(X^{l}, Y^{l}\right)$ value corresponding to each element $l$ of $L$.

(6) The final image quality assessment value MSMLD can be achieved with formula (4). Consider

$$
\operatorname{MSmLD}(X, Y)=\prod_{l=0}^{K} \operatorname{MLD}\left(X^{l}, Y^{l}\right) .
$$

3.4. Remote Sensing Image Compression Quality Assessment Verification. Considering ensuring the universality of test for remote sensing image compression, we randomly select 30 original gray remote sensing images (512-by-512 pixel) from the Internet as a test set, which has a great deal of important characteristics such as gray, contrast, image resolution, texture complexity, and the target distribution and covers a variety of commonly used scenes in remote sensing image, such as sea, mountain, airport, buildings, and motors. Some of the typical remote sensing images in the test set are shown in Figure 5. 


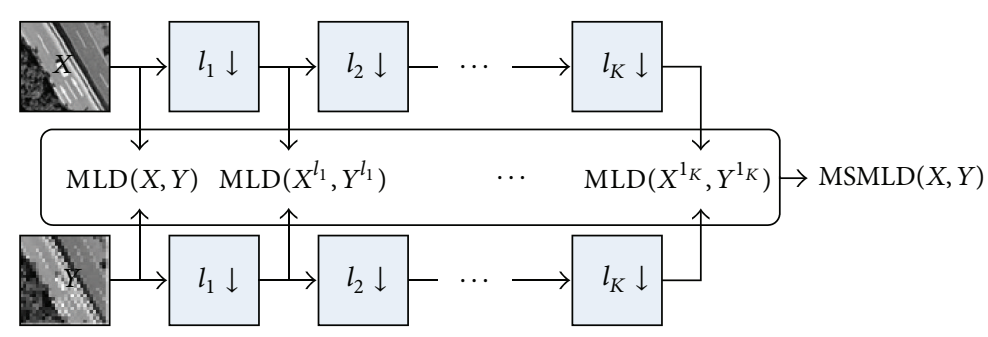

FIGURE 4: MSMLD calculation flow chart.

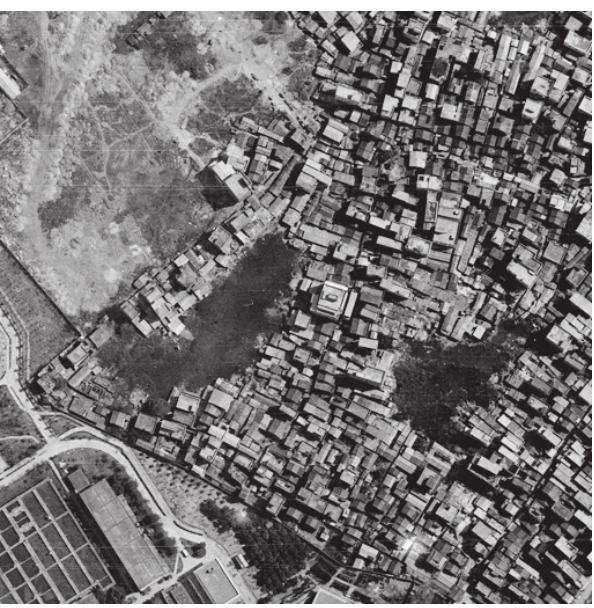

(a)

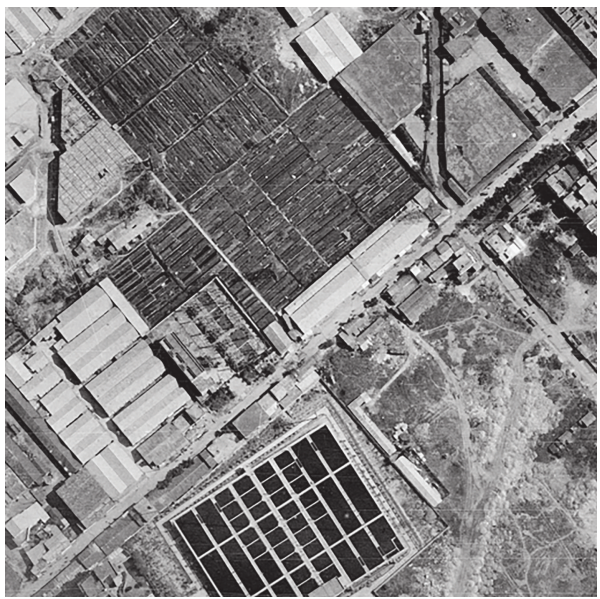

(c)

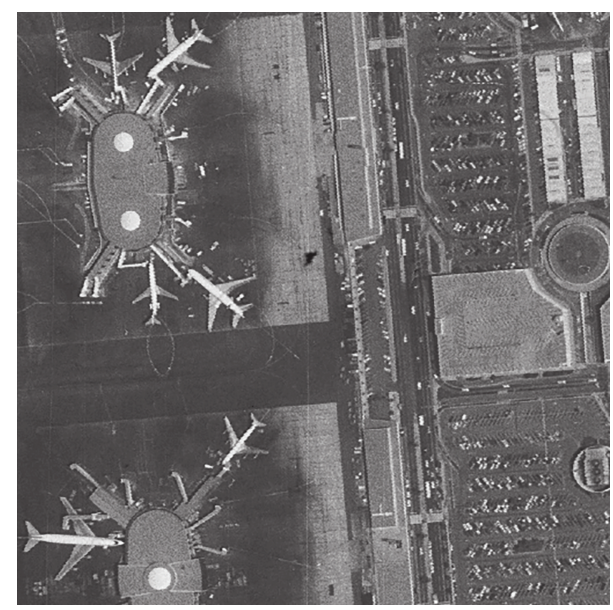

(b)

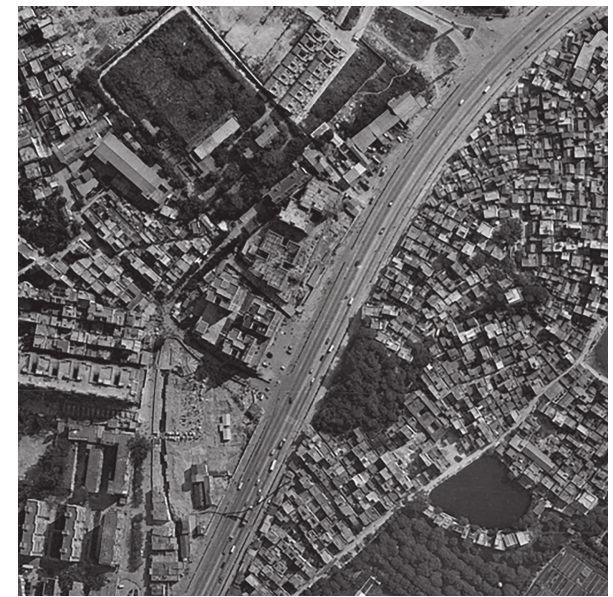

(d)

FIGURE 5: Some typical images (512-by-512 pixel) of the test database.

The distorted image is the decompressed image of the original image in test set. According to the analysis in Section 1, for covering various compression distortions, some of the most commonly used coding methods for remote sensing image such as JPEG2000 [5], JPEG [2], HD-PHOTO [26], BHC [27, 28], and FRACTAL [29] are chosen to generate distorted images with varying compression ratios. Finally a test image database is built with a total number of 750 images (30 images are compressed with 5 algorithms at 5 compression ratios separately). Some of the typical distorted images caused by different coding methods with various compression ratios are shown in Figures 6 and 7.

It is obvious that subjective scores are unstable and may be easily affected by many factors such as observation environment and individual capacity. Hence some rules of observing environment, scoring criteria, and score-processing methods are designed to avoid the random influences and ensure that the subjective scores are more close to human visual 


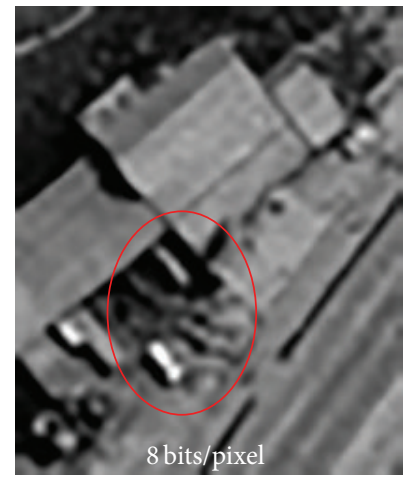

(a)

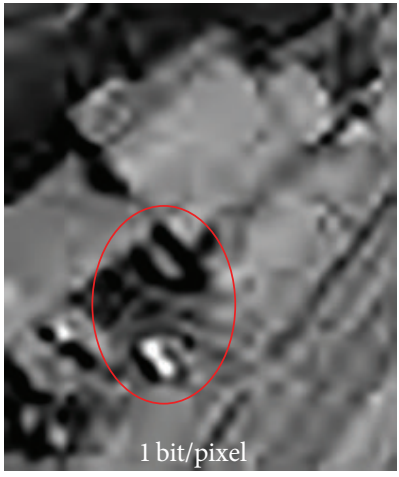

(b)

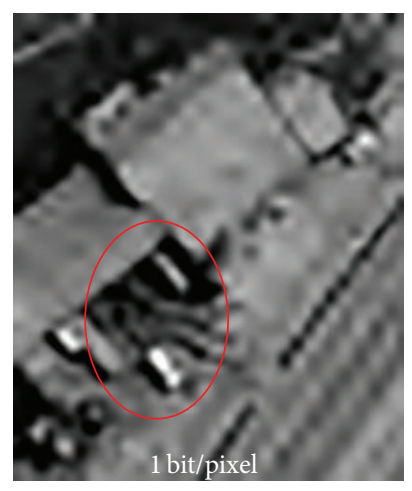

(c)

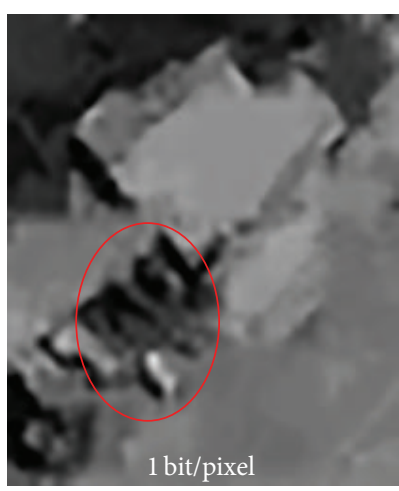

(d)

FIGURE 6: The distortions caused by the different compression methods with the same compression ratio. (a) A part (50-by-60 pixel) of an original eight-bit image. (b) JPEG compression at 1 bit per pixel. (c) BHC compression at 1 bit per pixel. (d) Fractal compression at 1 bit per pixel.

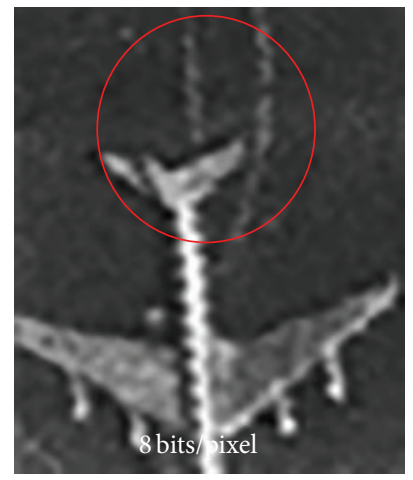

(a)

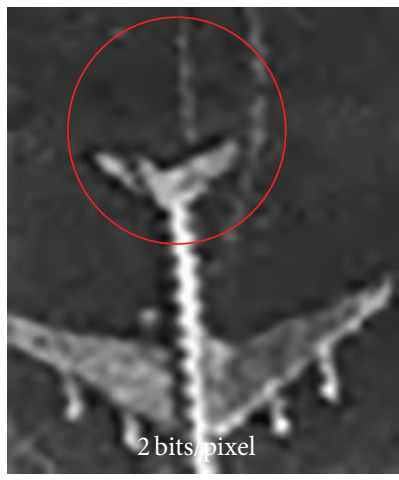

(b)

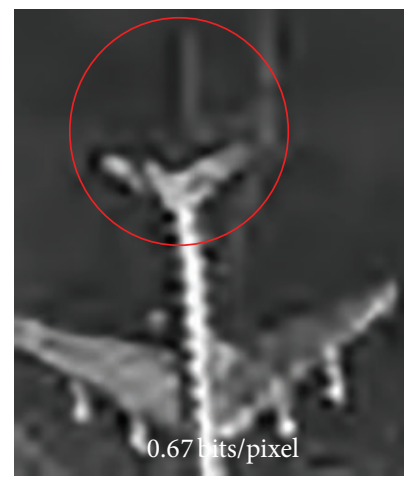

(c)

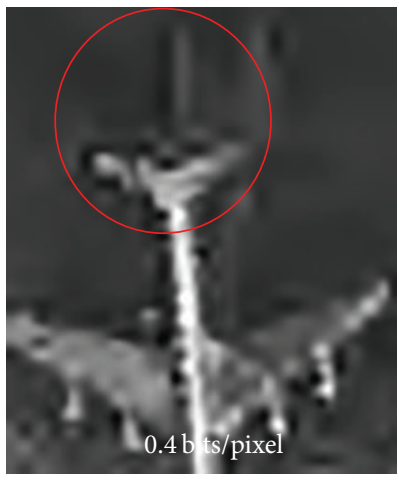

(d)

FIGURE 7: The distortions caused by JPEG2000 with different compression ratios. (a) A part (50-by-60 pixel) of an original eight-bit image. (b) JPEG2000 compression at 2 bpp. (c) JPEG2000 compression at 0.66 bpp. (d) JPEG2000 compression at 0.4 bpp.

perception. After that, subjective quality scores of distorted images are given, and the probability of unreasonable scoring is reduced by pairwise comparison and the distortion sorting. The final mean opinion score (MOS) of the distorted image is determined by the weighted evaluation scores from all interpreters.

The MSMLD algorithms are implemented and compared with state-of-the-art assessment methods such as PSNR [30], VSNR (visual signal-to-noise ratio) [16], WSNR [20], NQM [21], VIF [24], SSIM [22], and MS-SSIM [23]. The sorting of objective quality metrics is transformed to the predicted MOS score through nonlinear regression, denoted by MOSr, which is then compared with the actual MOS in the test image database. Nonlinear regression fitting function is selected from video quality expert group (VQEG II) [31] as

$$
\operatorname{MOS} r=b_{1} Q^{3}+b_{2} Q^{2}+b_{3} Q+b_{4} \text {, }
$$

where $Q$ is the original objective score, MOS $r$ is the mapping score, and $b_{1}, b_{2}, b_{3}$, and $b_{4}$ are parameters from VQEG II. Some correlation factors such as Pearson correlation coefficient (CC), Spearman rank order correlation coefficient
(SROCC), root mean square error (RMSE), and mean absolute error (MAE) are used to compare the MOSr and MOS. A higher score indicates a better performance of the objective assessment for CC and SROCC; conversely, a lower score is better for RMSE and MAE.

The correlation factors (such as CC, SROCC, RMSE, and MAE) of MOSr and MOS are calculated to quantify the assessing performance, and values are shown in Table 1. The results show that the metrics of VIF, MS-SSIM, and MSMLD can obtain a better consistency with MOS, benefiting from higher values in CC and SROCC and lower values in RMSE and MAE. However the metrics of NQM, PSNR, VSNR, and WSNR show a bad consistency with MOS, due to lower values in CC and SROCC and higher values in RMSE and MAE. All indicators show that MSMLD has the best consistency with MOS. The reason why MSMLD has a better consistency with MOS lies in the following three points. Firstly, the NQM, PSNR, VSNR, and WSNR metrics focus on pixel differences without considering contexture impacts. Secondly, VIF and MS-SSIM do not take into consideration the pixel-level distortions. Thirdly, the MSMLD takes into account the multilevel distortions (such as pixel-level, contexture-level, 


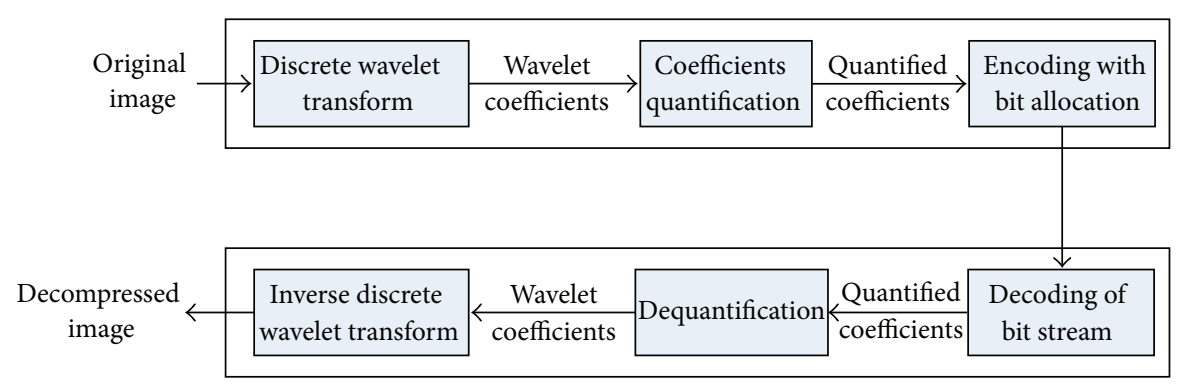

FIGURE 8: Basic scheme of DWT-based compression.

TABLE 1: Comprehensive performance of image quality evaluation methods.

\begin{tabular}{lcccc}
\hline Methods & CC & SROCC & RMSE & MAE \\
\hline NQM & 0.740578 & 0.737486 & 8.435576 & 8.904648 \\
PSNR & 0.758237 & 0.762692 & 7.124543 & 6.610797 \\
VSNR & 0.818448 & 0.809578 & 7.303455 & 5.625457 \\
WSNR & 0.832695 & 0.831679 & 7.051423 & 5.517978 \\
SSIM & 0.870765 & 0.870154 & 6.087862 & 4.805179 \\
VIF & 0.898674 & 0.898976 & 5.402187 & 4.166768 \\
MS-SSIM & 0.918475 & 0.922437 & 4.814498 & 3.527578 \\
MSMLD & $\mathbf{0 . 9 2 7 6 7 4}$ & $\mathbf{0 . 9 2 8 8 9 5}$ & $\mathbf{4 . 6 0 5 9 5 7}$ & $\mathbf{3 . 4 0 6 6 7 5}$ \\
\hline
\end{tabular}

and content-level) and gets a better property in accuracy and integrity of image quality assessment.

The proposed MSMLD provides a way which has better consistency with subjective perception values than current state-of-the-art methods in remote sensing image compression assessment. On the one hand, it can be used as an important tool for image quality assessment. On the other hand, it can also be applied to coding algorithm to predict compression quality. The latter will be discussed further in the following sections.

\section{Model of Compression Quality Prediction Based on MSMLD}

Although the proposed MSMLD has a better ability to assess image quality than other methods, this assessment is computationally heavy, since the evaluation of the compression quality is an expensive operation, which requires performing coding, decoding, and then comparison between original and decompressed images. Thus it is interesting to study a model which can epitomize the principle of MSMLD and can predict the compression quality at a preencoding stage or without coding. Later, the design and construction process of a quality prediction model will be introduced from three aspects. First, to choose an outstanding evaluation method and establish image data set for test, it has been done in the last section. Second, select some proper image characteristics (or functions) as predictive factors for DWTbased compression. Third, establish the relationship model between the evaluation results and predictive factors. Finally, apply the model to DWT-based coding algorithm to predict compression quality accurately.
4.1. Determination of Predictive Factors for DWT-Based Coding. In order to let a predictive model accurately reflect the compression quality of remote sensing images with DWTbased coding, it is necessary to analyze factors that affect compressing distortion. The basic diagram of DWT-based coding consists of three basic steps [12] as in Figure 8. First, DWT offers a highly efficient and flexible way to decompose and decorrelate the original image and bring about a new form in wavelet domain to support a more efficient coding. Second, the quantization step restricts wavelet coefficients into a limited series of values, that is, removes information considered to be redundant or useless. Meanwhile, the loss is generated in this irreversible process. Third, the encoding step assigns to each quantified coefficient a code as short as possible, and bits allocation is weighed and balanced on the different subbands of the image according to a given compression ratio (CR). The image decompression follows an inverse procedure.

The nature of an image histogram provides many clues to the character of the image. We have used a set of gray level image statistics and have analyzed various images to see if any particular image feature is mainly responsible for the variation in MSMLD values among various images. Therefore, a set of image characteristics that are commonly used in DWT-based methods are chosen as candidates to establish a quality prediction model of MSMLD, including image gray level $I_{l}$, image contrast $I_{c}$, and image activity measure $I_{a}$. They are defined as

$$
I_{l}=\frac{1}{W \times H} \sum_{j=1}^{H} \sum_{i=1}^{W} x_{i j},
$$


TABLE 2: Goodness-of-fit analysis between image characteristics and regression functions.

\begin{tabular}{lccccrr}
\hline & $I_{l}$ & $I_{c}$ & $I_{a}$ & $W_{c}$ & $W_{p}$ & 0.418 \\
\hline Linear & 0.000 & 0.071 & 0.730 & 0.702 & 0.802 & 0.378 \\
Logarithm & 0.000 & 0.060 & 0.839 & 0.799 & 0.899 \\
Quadratic & 0.030 & 0.077 & 0.838 & 0.759 & 0.332 & 0.898 \\
$S$ & 0.003 & 0.041 & 0.721 & 0.718 & 0.832 \\
Index & 0.000 & 0.071 & 0.749 & 0.718 & 0.432 \\
Logistic & 0.000 & 0.071 & 0.749 & & 0.894 \\
\hline
\end{tabular}

$$
\begin{aligned}
I_{c}= & \sqrt{\frac{1}{W \times H} \sum_{j=1}^{H} \sum_{i=1}^{W}\left(x_{i j}-I_{l}\right)^{2}}, \\
I_{a}= & \frac{1}{W \times(H-1)} \sum_{i=1}^{W} \sum_{j=1}^{H-1}\left|x_{i j}-x_{i(j+1)}\right| \\
& +\frac{1}{(W-1) \times H} \sum_{i=1}^{W-1} \sum_{j=1}^{H}\left|x_{i j}-x_{(i+1) j}\right|,
\end{aligned}
$$

where $W$ and $H$ are the width and height of the image, respectively, and $x_{i j}$ represent the luminance value of pixel in the image.

In addition, according to the characteristics of DWTbased compression algorithms, some special parameters about wavelet coefficients are selected as frequency domain characteristics of the image, including coefficients average $W_{c}$, coefficients energy concentration $W_{p}$, and bit-plane entropy average $W_{e}$. They are defined as

$$
\begin{aligned}
& W_{c}=\frac{1}{W \times H} \sum_{j=1}^{H} \sum_{i=1}^{W} c_{i j}, \\
& W_{p}=\frac{\left(64 \times \sum_{j=1}^{H / 8} \sum_{i=1}^{W / 8} \widetilde{c}_{i j}^{2}\right)}{\left(\sum_{j=1}^{H} \sum_{i=1}^{W} c_{i j}^{2}\right)}, \\
& W_{e}=\frac{1}{P} \sum_{k=1}^{P} B_{k},
\end{aligned}
$$

where $c_{i j}$ represents the wavelet coefficient values of image with DWT, $P$ represents the number of bit-planes, and $B_{k}$ represents the entropy of the bit-plane.

To establish a function between image characteristics and compression quality, 86 gray images are selected as the training set, in which about 70 percent of images are remote sensing images, and every one is a 512-by-512 pixel, eight bits per pixel. JPEG2000 is used to compress all images at different CR, such as $2.0 \mathrm{bpp}, 1.0 \mathrm{bpp}, 0.8 \mathrm{bpp}, 0.67 \mathrm{bpp}$, $0.5 \mathrm{bpp}$, and $0.4 \mathrm{bpp}$. A distorted image database is composed of all decompressed images with different CR. Then, quality assessment is applied to the distorted image database with MSMLD, and the objective evaluation values of images are achieved. In the end, a final training set comes into being, consisting of the original images, decompressed images, and the objective evaluation values.

In order to explore if any particular image feature is mainly responsible for the variation in MSMLD values among various images, a set of gray level image statistics are analyzed with scatter diagram [32] to determine whether the two variables are related, and image characteristic and objective evaluation value are plotted against each other as a scatter diagram. Figure 9 shows the relationship between each characteristic of images and image objective evaluation values at $0.5 \mathrm{bpp}$ with MSMLD. Experiments indicate that every characteristic has different scatterplots with image objective evaluation values, and $I_{l}$ and $I_{c}$ have less relevant degree with MSMLD values, but $I_{a}, W_{c}, W_{p}$, and $W_{e}$ link very well with MSMLD values.

In order to choose subsequent prediction factors more accurately from the above candidates, curvilinear regression analysis is further used to reveal interdependency between each characteristic itself and MSMLD values. SPSS data statistical software is used to process six kinds of curvilinear regression analysis, such as linear, logarithm, exponential, $S$ type curve, logistic, and quadratic polynomial. Table 2 is fitting results of the analysis. From the results, it can be made sure that $I_{l}, I_{c}, W_{p}$, and $W_{e}$ in quadratic form link well with MSMLD values, and $I_{a}$ and $W_{c}$ in logarithm form have better dependency with MSMLD values relatively. For this reason, the quadratic form of $I_{l}, I_{c}, W_{p}$, and $W_{e}$ and logarithm form of $I_{a}$ and $W_{c}$ are chosen as the optional factors for multiple linear regression analysis in the next steps. According to the above analysis, six factors such as $I_{l}^{2}, I_{c}^{2}, W_{p}^{2}, W_{e}^{2}, \ln ^{I_{a}}$, and $\ln ^{W_{c}}$ will be chosen as the optional factors of a predictive model for multiple linear regression analysis in the late steps.

4.2. Construction of Prediction Model. After determining candidate predictors, the final predictors should be selected to establish a quality prediction model. Multiple linear regression (MLR) [33] is a powerful tool commonly used to find a mathematical relationship between a group of random variables. The goal of MLR is to model the relationship between the explanatory and response variables, and the model can create a relationship in the form of a linear that best approximates all the individual data points. There are three techniques employed in MLR, such as forward selection, backward elimination, and stepwise regression. Stepwise regression is most frequently used in several procedures, and it proceeds with forward selection, but each time a variable is added, and a backward elimination step occurs to test whether any variables entered previously can be removed.

In view of image compressed at $0.5 \mathrm{bpp}$, the final predictors are selected and the coefficients of model are decided by MLR with stepwise regression. Table 3 shows the analysis 


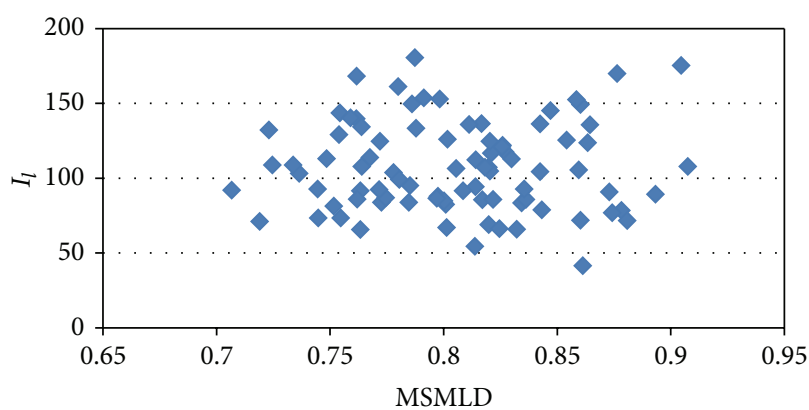

(a)

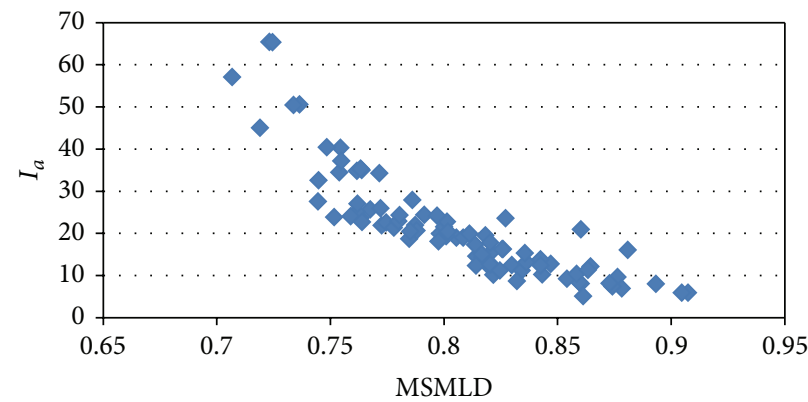

(c)

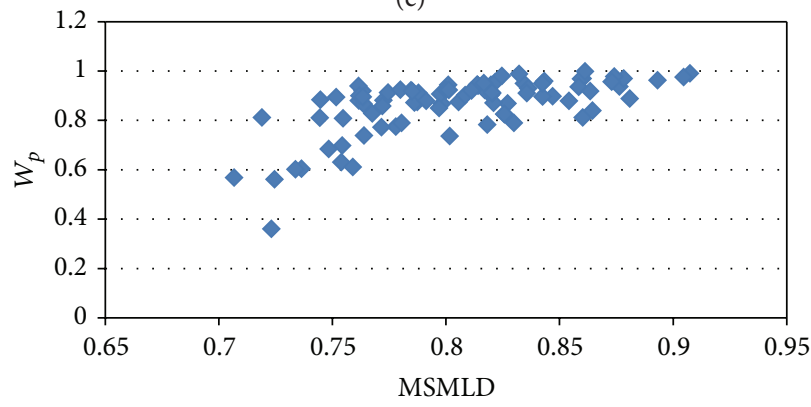

(e)

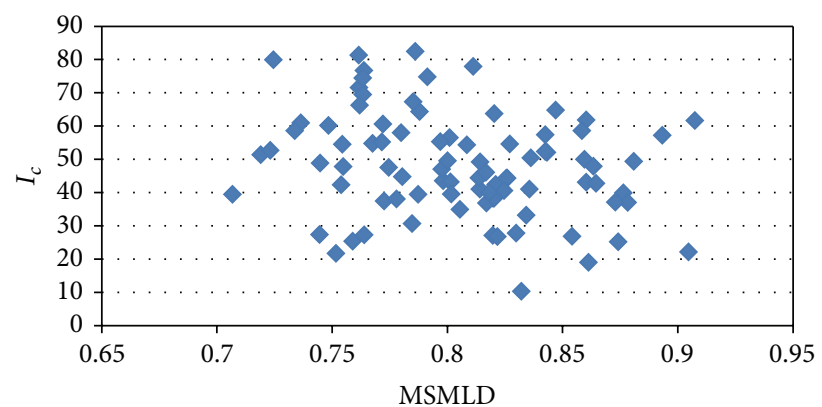

(b)

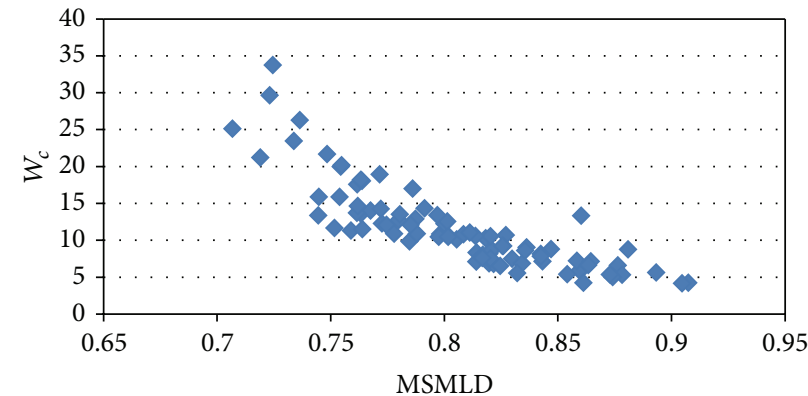

(d)

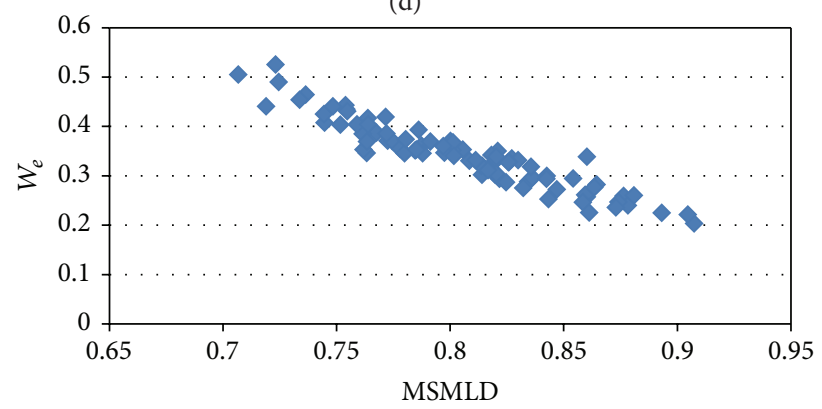

(f)

FIGURE 9: Scatter plot of feature values relative to distortion. (a) MSMLD versus $I_{l}$. (b) MSMLD versus $I_{c}$. (c) MSMLD versus $I_{a}$. (d) MSMLD versus $W_{c}$. (e) MSMLD versus $W_{p}$. (f) MSMLD versus $W_{e}$.

TABLE 3: Coefficients of predictors with multiple linear regression.

\begin{tabular}{|c|c|c|c|c|c|}
\hline \multirow{2}{*}{ Model } & \multicolumn{2}{|c|}{ Unstandardized coefficients } & \multirow{2}{*}{$\begin{array}{c}\text { Standardized coefficients } \\
\text { Beta }\end{array}$} & \multirow{2}{*}{$t$} & \multirow{2}{*}{ Sig. } \\
\hline & $B$ & Standard error & & & \\
\hline \multicolumn{6}{|l|}{1} \\
\hline (Constant) & 1.024 & 0.009 & & 119.589 & 0.000 \\
\hline$W_{e}^{2}$ & -0.638 & 0.025 & -0.943 & -25.993 & 0.000 \\
\hline \multicolumn{6}{|l|}{2} \\
\hline (Constant) & 1.129 & 0.022 & & 51.098 & 0.000 \\
\hline$W_{e}^{2}$ & -0.787 & 0.036 & -1.163 & -21.594 & 0.000 \\
\hline$W_{p}^{2}$ & -0.072 & 0.014 & -0.273 & -5.073 & 0.000 \\
\hline \multicolumn{6}{|l|}{3} \\
\hline (Constant) & 1.106 & 0.024 & & 46.995 & 0.000 \\
\hline$W_{e}^{2}$ & -0.775 & 0.036 & -1.146 & -21.650 & 0.000 \\
\hline$W_{p}^{2}$ & -0.063 & 0.014 & -0.242 & -4.476 & 0.000 \\
\hline$I_{l}^{2}$ & $1.171 E-4$ & 0.000 & 0.077 & 2.387 & 0.019 \\
\hline
\end{tabular}


results; the column of "model" indicates the count of "stepwise" in the overall analysis process and the selected factors in each cycle. The column of " $B$ " represents coefficient of each predictor in the model. The table illustrates that the stepwise regression analysis iterates 3 cycles and, respectively, selects $W_{p}^{2}$ and $W_{e}^{2}$ square and $I_{l}^{2}$ as predictive factors whose coefficients have been given.

Thus, for remote sensing images that are compressed at $0.5 \mathrm{bpp}$ with JPEG2000 algorithm, the predictive values of image quality assessment with MSMLD can be predicted comprehensively by three statistics: the mean value of image gray level $I_{l}$, coefficients energy concentration $W_{p}$, and bitplane entropy average $W_{e}$. The function of prediction model is shown as

$$
\begin{aligned}
P_{\text {mld-16 }}= & 1.106-0.775 \times W_{e}^{2} \\
& -0.063 \times W_{p}^{2}+0.0001171 \times I_{l}^{2} .
\end{aligned}
$$

In order to evaluate the accuracy of prediction models, residuals between the image quality evaluation values and predictive values are calculated and represented by the standardized residuals histogram shown in Figure 10. The histogram shows that the residuals between predictive values and actual values take on normal distribution, and the estimated standard deviation is defined as $\sigma$. The confidence level is referred to descript the probability where a confidence interval will contain given residuals; from Figure 10 we can see that a 95\% confidence level can be achieved with a confidence interval of $2 \sigma$, and a $70 \%$ confidence level can be achieved with a confidence interval of $\sigma$. It means the predicted values are in good correlation with the actual value and model predictive accuracy is perfectly excellent.

Similarly, prediction models of MSMLD can be constructed for CR of $0.8 \mathrm{bpp}$ and $0.67 \mathrm{bpp}$, and the value of MSMLD can also be predicted with $I_{l}, W_{p}$, and $W_{e}$ by the curve regression analysis and multiple linear regression. As a result, it shows that the joint function of the brightness of the image $I_{l}$, wavelet energy concentration $W_{p}$, and wavelet entropy value $W_{e}$ is suitable to be applied to any compression ratio to predict image quality, and the only thing that needs to be done is to adjust the coefficients of predictors according to the given CR.

Based on the above, a method of multiple linear regression analysis called "enter" is used to analyze the linear relationship between $I_{l}, W_{p}, W_{e}$ and image compression quality, and a prediction model is built as formula (9). The model is applied to JPEG2000 and BHC that is another prominent DWT-based coding method for remote sensing image and can accurately predict compression quality in various bit ratios, such as $2 \mathrm{bpp}, 1 \mathrm{bpp}, 0.8 \mathrm{bpp}, 0.67 \mathrm{bpp}, 0.5 \mathrm{bpp}$, and 0.4 bpp. Consider

$$
P_{\mathrm{mld}-r}=c_{r}+\alpha_{r} W_{e}^{2}+\beta_{r} W_{p}^{2}+\gamma_{r} I_{l}^{2}
$$

where

$$
\begin{aligned}
& \left(\begin{array}{llll}
c_{4} & \alpha_{4} & \beta_{4} & \gamma_{4} \\
c_{8} & \alpha_{8} & \beta_{8} & \gamma_{8} \\
c_{10} & \alpha_{10} & \beta_{10} & \gamma_{10} \\
c_{12} & \alpha_{12} & \beta_{12} & \gamma_{12} \\
c_{16} & \alpha_{16} & \beta_{16} & \gamma_{16} \\
c_{20} & \alpha_{20} & \beta_{20} & \gamma_{20}
\end{array}\right) \\
& =\left(\begin{array}{llll}
1.114 & -0.451 & -0.024 & 3.50 E-05 \\
1.157 & -0.717 & -0.061 & 9.36 E-05 \\
1.158 & -0.769 & -0.073 & 1.24 E-04 \\
1.132 & -0.759 & -0.067 & 1.30 E-04 \\
1.106 & -0.775 & -0.063 & 1.17 E-04 \\
1.088 & -0.794 & -0.069 & 1.68 E-04
\end{array}\right) .
\end{aligned}
$$

\section{Verification and Analysis}

In order to verify the accuracy of the proposed prediction model, 30 images are randomly selected from the Internet as a test set, which mostly are remote sensing images with different characteristics and resolution, 512-by-512 pixel size, and eight bits per pixel. All images of the test set are beyond the training set and are compressed by JPEG2000 method with different $\mathrm{CR}$, such as $2 \mathrm{bpp}, 1 \mathrm{bpp}, 0.8 \mathrm{bpp}, 0.67 \mathrm{bpp}$, $0.5 \mathrm{bpp}$, and $0.4 \mathrm{bpp}$. In the end, a final test set including 180 decompressed images is established.

For each image of test set, the quality assessment value of decompressed image is calculated by MSMLD as the actual result, and the quality assessment value is calculated by the preferred prediction model as the predictive result. Two typical results are shown in Figure 11.

All differences between the actual results and the predictive results are computed and shown as cumulative distribution function (CDF) in Figure 12. According to the figure, on the one hand, most of the absolute values of the differences are less than 0.02 and are approximately distributed as normal, concentrating around 0 . On the other hand, the error distribution is more concentrated with lower $\mathrm{CR}$, and the predictive accuracy decreases with the increase of the compression ratio in a way; nevertheless, all errors are not more than 0.03 as compression ratios are $1 \mathrm{bpp}, 0.8 \mathrm{bpp}$, $0.67 \mathrm{bpp}$, and $0.5 \mathrm{bpp}$; only three of the errors are greater than 0.03 as compression ratio is $0.4 \mathrm{bpp}$.

In addition, the prediction errors in various compression ratios are counted up and shown in Table 4. According to the table, $98.33 \%$ of the prediction errors are less than 0.03 , and $89.44 \%$ of the prediction errors are less than 0.02 . Meanwhile, the prediction model can achieve relatively higher prediction accuracy for lower compression ratio; for example, $96.66 \%$ of the prediction errors are less than 0.02 when the compression ratio is lower than 2 bits per pixel. Due to the range of evaluation values of MSMLD which is $[0,1]$, magnitude error value from 0.02 to 0.03 accounts for only $2 \%$ to $3 \%$ of the entire range, so the proposed prediction model can accurately predict the assessment of image quality.

As previously described, at present the methods used for image compression quality prediction are mainly some traditional methods, such as PSNR. Since their range of 


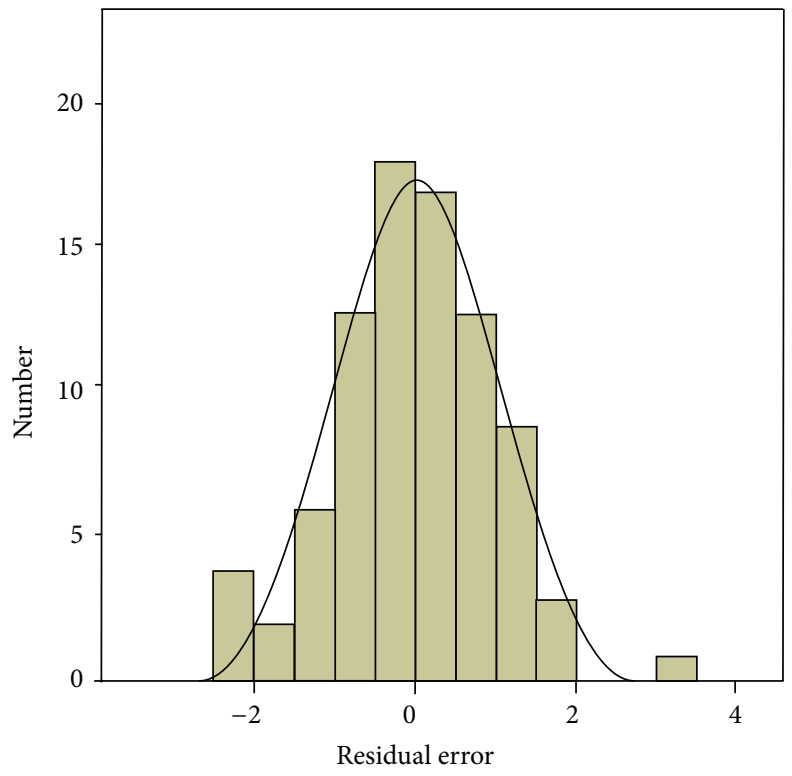

FIGURE 10: Histogram of standard residuals.
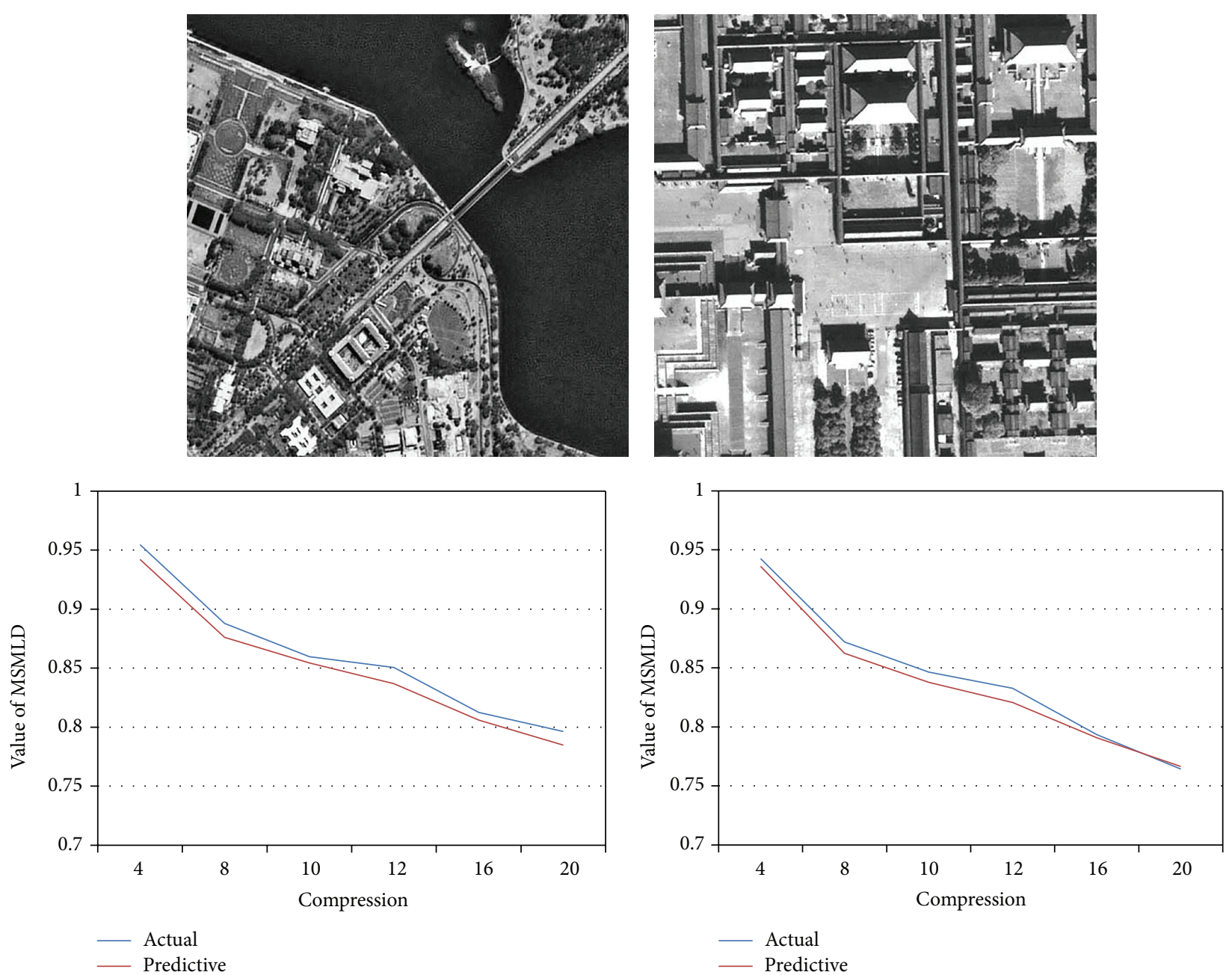

FIGURE 11: Partial comparison of the actual with the predictive MSMLD values. 


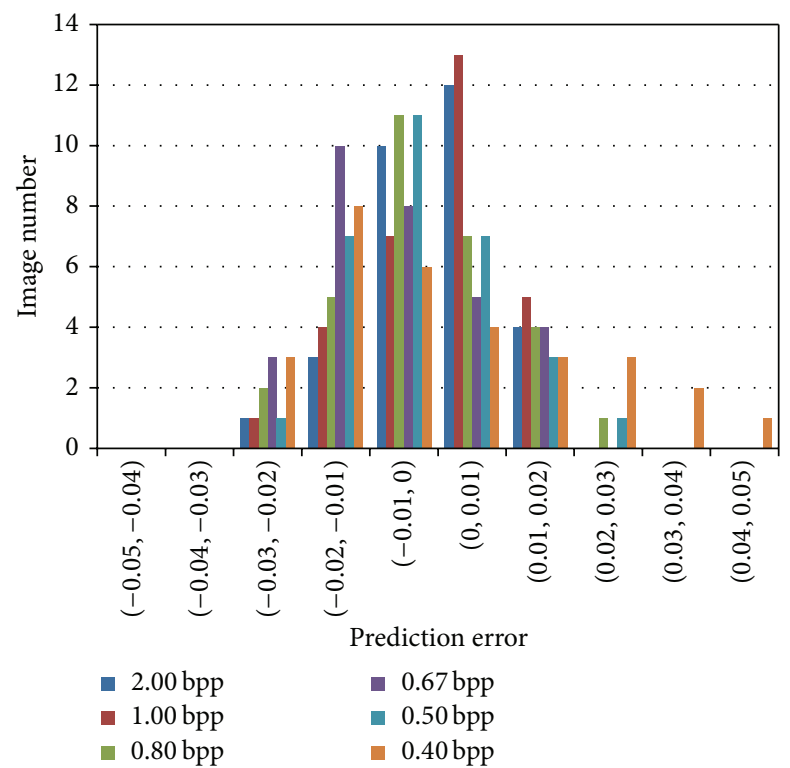

Figure 12: Cumulative histogram of prediction error. TABLE 4: Percentage of prediction error distribution.

\begin{tabular}{lccc}
\hline Compression ratio (bits per pixel) & Error less than 0.02 & Error less than 0.03 & Error more than 0.03 \\
\hline 2.00 & $96.67 \%$ & $100 \%$ & 0 \\
1.00 & $96.67 \%$ & $100 \%$ & 0 \\
0.80 & $90.00 \%$ & $100 \%$ & 0 \\
0.670 & $90.00 \%$ & $100 \%$ & 0 \\
0.50 & $93.33 \%$ & $100 \%$ & 0 \\
0.40 & $70.00 \%$ & $90.00 \%$ & $10.00 \%$ \\
\hline Average & $89.44 \%$ & $98.33 \%$ & $1.67 \%$
\end{tabular}

the quality evaluation values is different from MSMLD, it is difficult to compare prediction results directly. Therefore, percentage of the prediction error and the actual value are calculated as an indicator of comparison. For example, with CR of $0.5 \mathrm{bpp}$, the predictive value of PSNR is calculated according to the prediction model in [11]; then the actual PSNR and the absolute error between the predicted value and the actual value are also calculated. Finally, the percentage of the prediction error and the actual value are obtained. In the same way, percentage of the prediction error can also be calculated with the proposed MSMLD, and all results are shown in Table 5. From the table, the average of the relative prediction errors is $1.54 \%$ by the predictive model in [11], and the average of the relative prediction errors is $1.09 \%$ by the proposed model of MSMLD. Obviously, the proposed model is of relatively higher prediction accuracy. Similar experiments are also used to test the BHC algorithm, just like on JPEG2000; the model can also predict the image compression quality of MSMLD accurately.

Meanwhile, the proposed MSMLD algorithm has better consistency with subjective perception values than PSNR in remote sensing image compression assessment, and the objective assessment results can show the distortion features and visual quality of compressed image well. Therefore, the conclusion can draw that the proposed prediction model of
MSMLD is not only accurate for assessment prediction, but also closer to the subjective evaluation of compressed image distortion.

\section{Conclusions and Future Work}

In the paper a novel image quality assessment method named MSMLD is preferred for remote sensing image compression; it is incorporating merits of pixel-level distortion, contexture-level distortion, content-level distortion, and multiscale structural similarity. Compared with stateof-the-art image quality assessment approaches like SSIM, VIF, PSNR, and so forth, the proposed MSMLD algorithm has a better consistency with subjective perception values than the current state-of-the-art methods in remote sensing image compression assessment, and the objective assessment results can show the distortion features and visual quality of compressed image well.

Basing on the above, by analyzing the correlation between multilevel differences and image characteristics, six important characteristics of image in space domain and frequency domain are preferred, such as image gray level, image contrast, image activity measure, wavelet coefficients average, wavelet coefficients energy concentration, and bit-plane entropy average. Furthermore, three characteristics that are 
TABLE 5: Relative error of prediction with CR of $0.5 \mathrm{bpp}$.

\begin{tabular}{|c|c|c|c|c|c|c|c|c|c|c|}
\hline Image number & 1 & 2 & 3 & 4 & 5 & 6 & 7 & 8 & 9 & 10 \\
\hline \multicolumn{11}{|c|}{ Percentage of relative errors } \\
\hline PSNR & 0.95 & 0.98 & 0.41 & 2.73 & 0.84 & 1.57 & 1.36 & 0.31 & 1.00 & 2.27 \\
\hline MSMLD & 2.54 & 0.35 & 1.58 & 0.58 & 1.23 & 1.57 & 2.96 & 0.07 & 1.74 & 1.65 \\
\hline Image number & 11 & 12 & 13 & 14 & 15 & 16 & 17 & 18 & 19 & 20 \\
\hline \multicolumn{11}{|c|}{ Percentage of relative errors } \\
\hline PSNR & 0.85 & 5.98 & 0.83 & 1.13 & 1.21 & 0.65 & 1.90 & 0.49 & 0.77 & 2.95 \\
\hline MSMLD & 1.68 & 0.61 & 0.82 & 0.02 & 0.74 & 0.21 & 0.06 & 0.06 & 0.72 & 0.62 \\
\hline Image number & 21 & 22 & 23 & 24 & 25 & 26 & 27 & 28 & 29 & 30 \\
\hline \multicolumn{11}{|c|}{ Percentage of relative errors } \\
\hline PSNR & 0.14 & 1.65 & 0.79 & 0.17 & 0.41 & 4.36 & 0.64 & 5.22 & 1.67 & 1.90 \\
\hline MSMLD & 1.17 & 2.42 & 1.65 & 2.17 & 0.97 & 1.18 & 0.90 & 1.36 & 1.02 & 0.20 \\
\hline
\end{tabular}

closely related to the quality assessment of MSMLD are chosen with multiple linear regression and used to establish a quality prediction model of MSMLD for the DWT-based coding algorithm. The quality prediction model is applied to a DWT-based compression algorithm named JPEG2000 and $\mathrm{BHC}$ for various compression ratios and tested with experiments; the experimental results show that the prediction accuracy of the proposed model is up to $98.33 \%$, and its mean prediction error is less than the current state-of-theart methods. It can predict the distortion of remote sensing image compression in multiple fixed bit rates efficiently.

Although the proposed prediction model of MSMLD can reflect the visual quality of remote sensing compressed images accurately, there are still some issues that deserve to be further investigated in the future. Firstly, besides the currently used low-level image visual features such as small target, complex edge texture, and uneven distribution of image content, there are some additional elements and characteristics of remote sensing images that may be taken into account further and be mapped into multilevel spatial semantics via visual feature extraction. Moreover, some highlevel semantics like object-based image classification and spatial relationship inference $[34,35]$ can also be introduced to forecast the image quality during remote sensing image compression. Secondly, some new concepts and opinions may be beneficial for the prediction of remote sensing image compression assessment, like image sketch [36] and image epitome [37], which could be derived from original images to assist the vision technologies and the compression assessment as well. At last, since the MSMLD is a full-reference method which requires full information of the reference image, it could be a serious impediment for some applications. So developing a reduced-reference prediction method based on MSMLD is essential in the future to reduce prediction complexity of image assessment with limited information of the compressed images.

\section{Conflict of Interests}

The authors declare that there is no conflict of interests regarding the publication of this paper.

\section{Acknowledgments}

This work is partially supported by the 973 Program (no. 2010CB327900), the NSFC (no. 61272347), and the National Science Fund for Distinguished Young Scholars (no. 61125206).

\section{References}

[1] B. Penna, T. Tillo, E. Magli, and G. Olmo, "Transform coding techniques for lossy hyperspectral data compression," IEEE Transactions on Geoscience and Remote Sensing, vol. 45, no. 5, pp. 1408-1421, 2007.

[2] "Information technology-digital compression and coding of continuous-tone still images," Tech. Rep. ISO/IEC 10918-1 and ITU-T Rec. T.81, 1992.

[3] P. Hou, M. Petrou, C. Underwood, and A. H. Hojjatoleslami, "Improving JPEG performance in conjunction with cloud editing for remote sensing applications," IEEE Transactions on Geoscience and Remote Sensing, vol. 38, no. 1, pp. 515-524, 2000.

[4] G. Yu, T. Vladimirova, and M. N. Sweeting, "Image compression systems on board satellites," Acta Astronautica, vol. 64, no. 9-10, pp. 988-1005, 2009.

[5] "Information technology-JPEG2000 image coding system," ISO/IEC 15444-1 and ITU-T Rec. T.800, 2000.

[6] D. Taubman and M. Marcellin, JPEG2000: Image Compression Fundamentals, Standards and Practice, Kluwer Academic, Norwell, Mass, USA, 2001.

[7] M. Rabbani and R. Joshi, "An overview of the JPEG 2000 still image compression standard," Signal Processing: Image Communication, vol. 17, no. 1, pp. 3-48, 2002.

[8] L. Pesquer, X. Pons, A. Cortes, and I. Serral, "Spatial pattern alterations from JPEG2000 lossy compression of remote sensing images: massive variogram analysis in high performance computing," Journal of Applied Remote Sensing, vol. 7, no. 1, Article ID 073595, 2013.

[9] Y. Li, R. Yan, C. Wu, K. Wang, S. Li, and Y. Wang, "Adaptive compression of remote sensing stereo image pairs," Journal of Applied Remote Sensing, vol. 4, no. 1, Article ID 041777, 2010.

[10] Image Data Compression, Recommendation for space data system standards, CCSDS 122.0-B-1, 2005.

[11] L. Li and Z. S. Wang, "Compression quality prediction model for JPEG2000," IEEE Transactions on Image Processing, vol. 19, no. 2, pp. 384-398, 2010. 
[12] A. Kourzi, D. Nuzillard, G. Millon, and F. Nicolier, "Quality estimation in wavelet image coding," in Proceedings of the13th European Signal Processing Conference (EUSIPCO '05), pp. 12271230, Antalya, Turkey, September 2005.

[13] S. Saha and R. Vemuri, "An analysis on the effect of image features on lossy coding performance," IEEE Signal Processing Letters, vol. 7, pp. 104-107, 2000.

[14] H. Koumaras, T. Pliakas, and A. Kourtis, "A novel method for pre-encoding video quality prediction," in Proceedings of the 16th IST Mobile and Wireless Communications Summit, pp. 1-4, Budapest, Hungary, July 2007.

[15] G. Wei, H. Jiang, and R. Yang, "Linear-regression model based wavelet filter evaluation for image compression," in Proceedings of the Asia-Pacific Conference on Wearable Computing Systems (APWCS '10), pp. 315-318, April 2010.

[16] D. M. Chandler and S. S. Hemami, "VSNR: a wavelet-based visual signal-to-noise ratio for natural images," IEEE Transactions on Image Processing, vol. 16, no. 9, pp. 2284-2298, 2007.

[17] S. Nichols, K. Hyunju, A. A. Humos, and H. J. Cho, "A performance evaluation on DCT and wavelet-based compression methods for remote sensing images based on image content," in Proceedings of the 17th International Conference on Geoinformatics (Geoinformatics '09), pp. 1-5, Fairfax, Va, USA, August 2009.

[18] M. Datcu, G. Schwarz, K. Schmidt, and C. Reck, "Quality evaluation of compressed optical and SAR images: JPEG vs. wavelets," in Proceedings of the International Geoscience and Remote Sensing Symposium (IGARSS '95), pp. 1687-1689, July 1995.

[19] L. Zhai, T. Xinming, and L. Lin, "Effects of JPEG2000 compression on remote sensing image quality," in Proceedings of the IEEE International Geoscience and Remote Sensing Symposium (IGARSS '06), pp. 3297-3300, August 2006.

[20] T. Mitsa and K. L. Varkur, "Evaluation of contrast sensitivity functions for the formulation of quality measures incorporated in halftoning algorithms," in Proceedings of IEEE International Conference on Acoustics, Speech and Signal Processing, pp. V301-V-304, Minneapolis, Minn, USA, April 1993.

[21] N. Damera-Venkata, T. D. Kite, W. S. Geisler, B. L. Evans, and A. C. Bovik, "Image quality assessment based on a degradation model," IEEE Transactions on Image Processing, vol. 9, no. 4, pp. 636-650, 2000.

[22] Z. Wang, A. C. Bovik, H. R. Sheikh, and E. P. Simoncelli, "Image quality assessment: from error visibility to structural similarity," IEEE Transactions on Image Processing, vol. 13, no. 4, pp. 600$612,2004$.

[23] Z. Wang, E. P. Simoncelli, and A. C. Bovik, "Multi-scale structural similarity for image quality assessment," in Proceedings of the 37th Asilomar Conference on Signals, Systems and Computers, pp. 1398-1402, Pacific Grove, Calif, USA, November 2003.

[24] H. R. Sheikh and A. C. Bovik, "Image information and visual quality," IEEE Transactions on Image Processing, vol. 15, no. 2, pp. 430-444, 2006.

[25] J. Yang, "Multiple description wavelet-based image coding using iterated function system," Mathematical Problems in Engineering, vol. 2013, Article ID 924274, 12 pages, 2013.

[26] Information technology-JPEG XR image coding system, ISO/IEC 29199-5 and ITU-T Rec. T.835, 2010.

[27] B. Li, R. Yang, and H. X. Jiang, "Remote-sensing image compression using two-dimensional oriented wavelet transform,"
IEEE Transactions on Geoscience and Remote Sensing, vol. 49, no. 1, pp. 236-250, 2011.

[28] R. H. Jiao, High efficient prediction coding and its optimization in image compression [Ph.D. dissertation], Beihang University, Beijing, China, 2008.

[29] Y. Fisher, Fractal Image Compression: Theory and Application, Springer, New York, NY, USA, 1995.

[30] H. R. Sheikh, M. F. Sabir, and A. C. Bovik, "A statistical evaluation of recent full reference image quality assessment algorithms," IEEE Transactions on Image Processing, vol. 15, no. 11, pp. 3440-3451, 2006.

[31] D. Djaenudin, H. Marwan, H. Subagjo, and A. Hidayat, Tech. Rep., Technical Guidance of Land Evaluation for Agricultural Commodities, Soil Research Institute, Agricultural Research and Development Agency, 2003.

[32] S. J. Maas and N. Rajan, "Normalizing and converting image DC data using scatter plot matching," International Journal of Remote Sensing, vol. 2, no. 7, pp. 1644-1661, 2010.

[33] L. S. Aiken, S. G. West, and S. C. Pitts, "Multiple linear regression," in Handbook of Psychology, 2003.

[34] M. Wang, T. Song, H. R. Sheikh et al., "Remote sensing image retrieval by scene semantic matching," IEEE Transactions on Geoscience and Remote Sensing, vol. 51, pp. 2874-2886, 2013.

[35] H. A. Jalab and N. A. Abdullah, "Content-based image retrieval based on electromagnetism-like mechanism," Mathematical Problems in Engineering, vol. 2013, Article ID 782519, 10 pages, 2013.

[36] R. Hu and J. Collomosse, "A performance evaluation of gradient field HOG descriptor for sketch based image retrieval," Computer Vision and Image Understanding, vol. 117, no. 7, pp. 790806, 2013.

[37] L. Benoit, J. Mairal, F. Bach, and J. Ponce, "Sparse image representation with epitomes," in Proceedings of the IEEE Conference on Computer Vision and Pattern Recognition (CVPR '11), pp. 2913-2920, Providence, RI, USA, June 2011. 


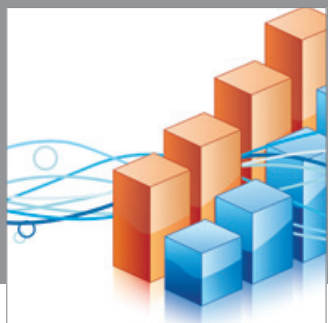

Advances in

Operations Research

mansans

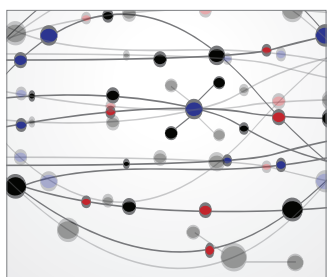

The Scientific World Journal
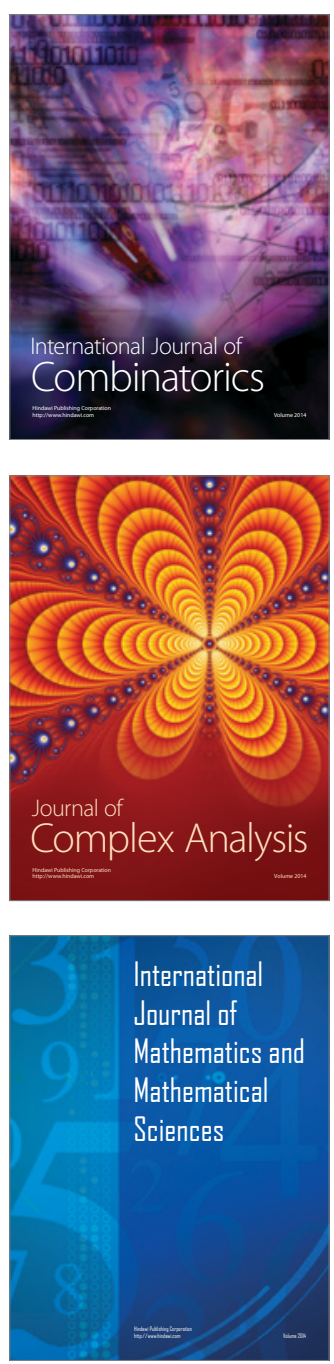
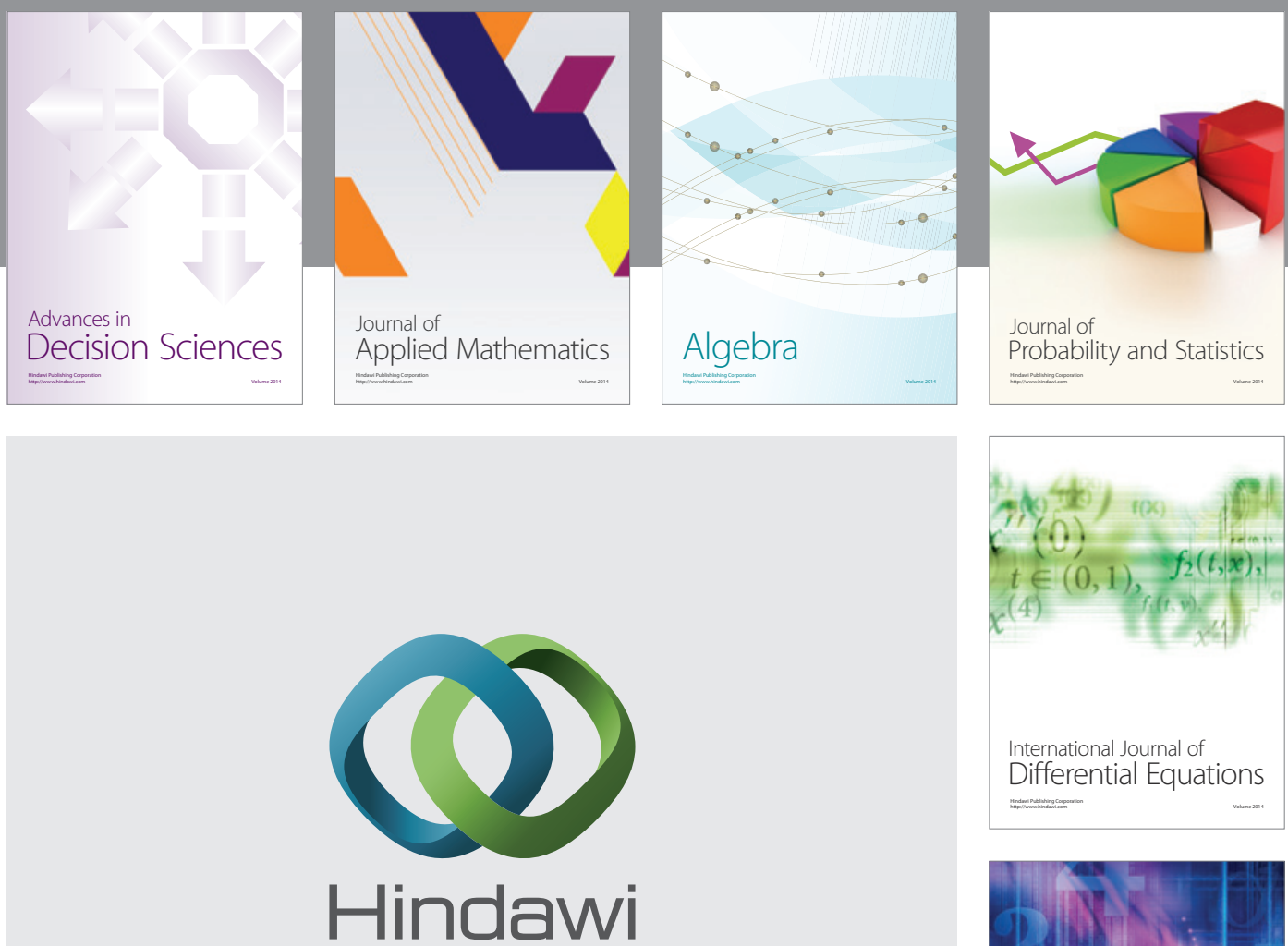

Submit your manuscripts at http://www.hindawi.com
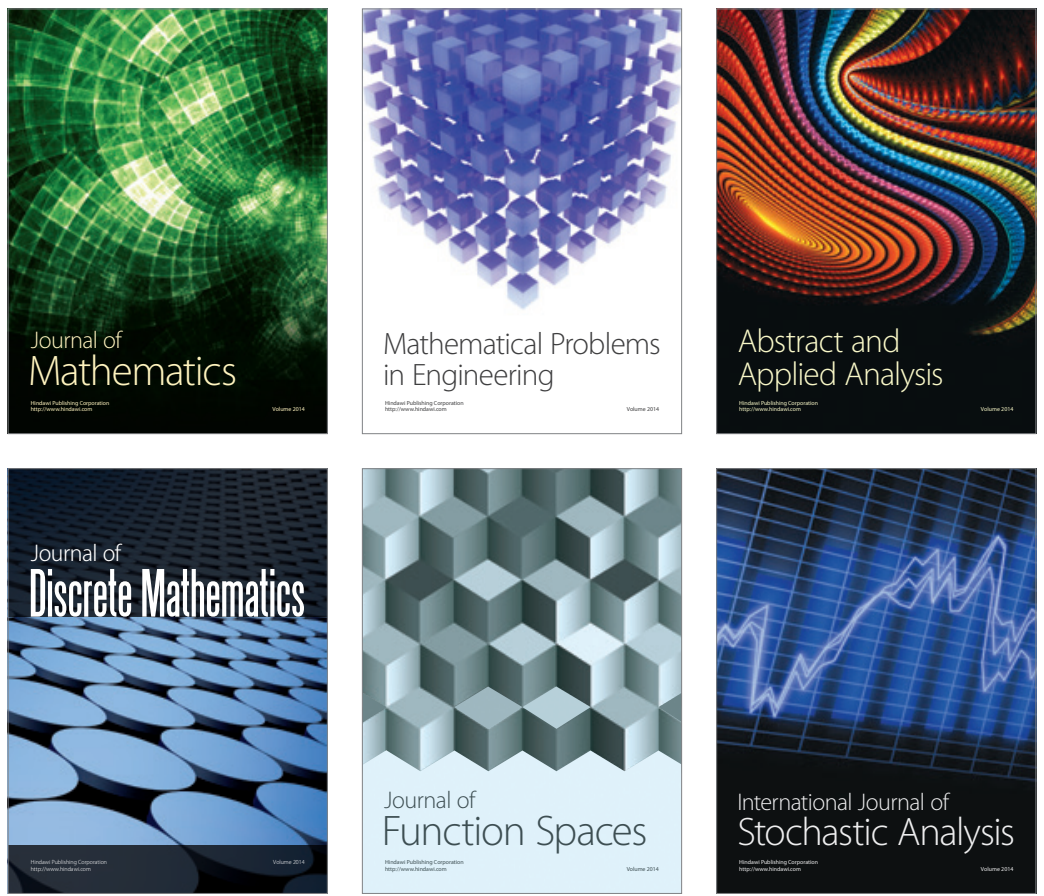

Journal of

Function Spaces

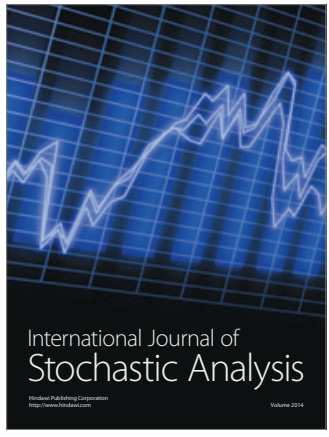

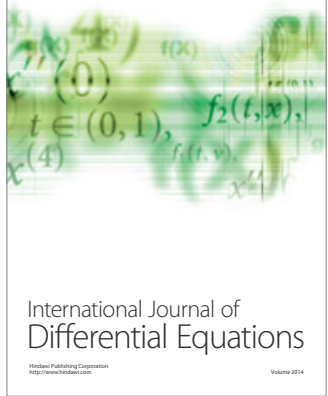
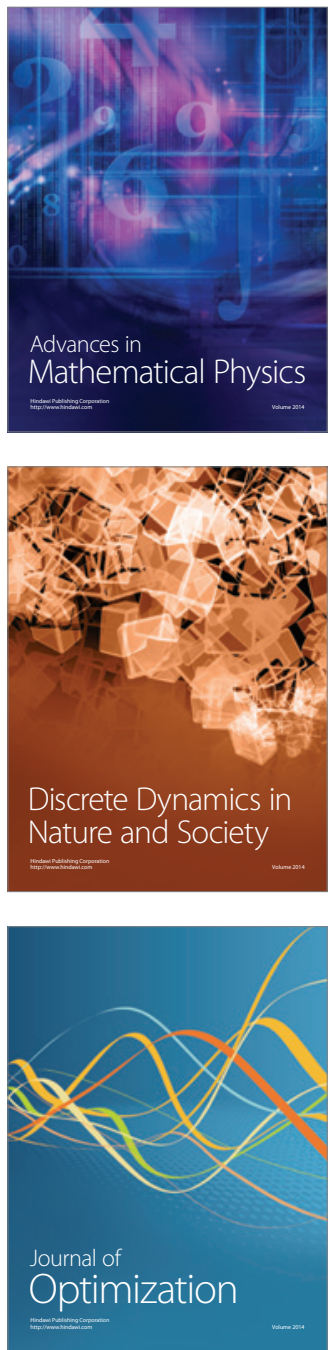